\title{
Association of Multiple Rib Fractures With Pneumonia After Successful Cardiopulmonary Resuscitation: A Retrospective Cohort Study Using Propensity Score Matching
}

\section{Yasuyuki Kawai ( $\boldsymbol{\nabla}$ k6k6k@naramed-u.ac.jp )}

Department of Emergency and Critical Care Medicine, Nara Medical University Shijo-cho, 840 Kashihara City, Nara, 6348522, Japan https://orcid.org/0000-0003-3630-6135

\section{Keisuke Takano \\ Nara Medical University \\ Keita Miyazaki \\ Nara Medical University \\ Koji Yamamoto \\ Nara Medical University \\ Yusuke Tada \\ Nara Medical University \\ Hideki Asai \\ Nara Medical University \\ Naoki Maegawa \\ Nara Medical University \\ Yasuyuki Urisono \\ Nara Medical University \\ Keigo Saeki \\ Nara Medical University \\ Hidetada Fukushima \\ Nara Medical University}

\section{Research}

Keywords: out of hospital cardiac arrest, cardiopulmonary resuscitation, pneumonia, rib fractures

Posted Date: November 2nd, 2020

DOI: https://doi.org/10.21203/rs.3.rs-99743/v1 
License: (c) (i) This work is licensed under a Creative Commons Attribution 4.0 International License. Read Full License 


\section{Abstract}

Background: Few studies have examined the impact of chest wall injury on respiratory complications after cardiopulmonary resuscitation. This is due to many confounding factors for the development of complications after cardiopulmonary resuscitation. Accordingly, we investigated the association between multiple rib fractures and the incidence of pneumonia during the post-resuscitation period after adjusting for confounding factors using a propensity score.

Methods: This single-centre, retrospective cohort study enrolled adult, non-traumatic, out-of-hospital, cardiac arrest patients who maintained circulation for $>48$ h between June 2015 and May 2019. Rib fractures were evaluated by computed tomography on the day of hospital admission, and the association with newly developed pneumonia within 7 days of hospitalisation was analysed using propensity score matching with adjustment for variables previously reported to be risk factors for the development of pneumonia.

Results: Of the 683 out-of-hospital cardiac arrest patients treated during the study period, 87 eligible cases were enrolled for analysis. Thirty-two patients had multiple rib fractures identified by computed tomography and 35 patients developed pneumonia. The presence of multiple rib fractures was significantly associated with a higher incidence of pneumonia (propensity score-adjusted hazard ratio: $3.51 ; 95 \%$ confidence interval: $1.59-7.72 ; p=0.002)$. Consistently, after propensity score matching, the multiple rib fracture group showed significantly shorter pneumonia-free survival than the non-multiple rib fracture group $(p<0.01)$.

Conclusion: Multiple rib fractures are independently associated with the development of pneumonia after successful resuscitation.

\section{Background}

Cardiac arrest (CA) is a leading cause of death in many countries. Successful resuscitation relies on immediate chest compression [1, 2]. Cardiopulmonary resuscitation (CPR), involving a high-flow fraction of hard and fast chest compressions, is associated with favorable neurological outcomes [3, 4]. However, several investigators pointed out that chest compressions can cause various complications $[5,6]$. The most common complication is rib fracture, and various studies have reported an increase in its frequency as guidelines have been revised and hard and fast chest compressions emphasised [6-8].

During the post-resuscitation period, patients develop a systemic inflammatory condition defined as postCA syndrome, during which time they are highly susceptible to infection [9-11] and generally develop pneumonia $[9,12,13]$. Previous studies indicated that pneumonia is common following out-of-hospital CA (OHCA) [10, 12], with a reported frequency of 29-70\% [9]. Several factors, including mechanical ventilation and therapeutic hypothermia, have been associated with the development of pneumonia among resuscitated CA patients [9-15]. It has been reported that rib fracture increases the frequency of pneumonia and worsens the prognosis, while surgical interventions on the chest wall improve prognosis 
among patients with blunt chest trauma [16]. However, the association of rib fracture and pneumonia in patients with CA during the post-resuscitation period has not been investigated.

We hypothesised that multiple rib fractures after resuscitation may be an independent risk factor for the development of pneumonia during the post-resuscitation period. However, it is difficult to accumulate samples because of the frequent updates (every 5 years) in the guideline recommendations regarding chest compression. Moreover, the incidence of rib fractures and pneumonia can vary between the guideline periods and institutions. This limits the variables that can be adjusted by multivariate analysis. To solve this problem, we investigated its causality among post-resuscitated patients by using the propensity score as a representative of known factors associated with the development of pneumonia.

\section{Methods}

\section{Study design}

This single-centre, retrospective, observational study was conducted to investigate the association between CPR-related rib fractures and the development of pneumonia during the post-resuscitation period. The study was approved by the institutional ethics committee of Nara Medical University, Nara, Japan.

\section{Data collection}

We investigated patients with OHCA admitted to our facility between June 2015 and May 2019. Data were obtained from hospital data sets recorded in the Utstein style [17]. The inclusion criteria were: age $\geq$ 18 years; diagnosis of non-traumatic CA; and achievement of sustained spontaneous circulation or extracorporeal circulation for $>48 \mathrm{~h}$. The exclusion criteria were: confirmed pre-existing pneumonia by chest computed tomography (CT) on hospital arrival; lack of chest CT evaluation after hospital admission; and missing data regarding time events for CPR.

We collected characteristics and the potential risk factors associated with pneumonia after CPR, including age $\geq 75$ years, sex, total CPR time (including bystander CPR), therapeutic hypothermia (TH), use of muscle relaxants, use of prophylactic antibiotics within $24 \mathrm{~h}$ of CA, use of mechanical ventilation, and number of rib fractures.

\section{Diagnosis}

Rib fracture was diagnosed through chest CT performed before admission to the intensive care unit. All CT images were acquired with a 64-row helical CT system (Optima CT660; GE Healthcare, Waukesla, WI, USA). The scan variables were as follows: $120 \mathrm{kVp}$; auto $\mathrm{mA}$; rotation time, $0.5 \mathrm{~s}$; helical pitch, 0.531 ; image noise, SD10. Coronal, sagittal, and three-dimensional reconstructed images were captured and used for diagnosis. We included all types of rib fractures: with or without displacement, incomplete, and Buckle [18] type. Previous studies reported that most rib fractures after resuscitation were bilateral and multiple [18-21]; thus, we defined three or more rib fractures as multiple rib fractures. 
We evaluated the development of pneumonia during the first 7 days of hospitalisation [13]. According to commonly used diagnostic criteria [22], pneumonia was diagnosed by the presence of clinically relevant findings at auscultation, decreased oxygenation that could not be explained by pulmonary oedema or atelectasis, new consolidation on a chest radiograph persistent for $\geq 48 \mathrm{~h}$, and an increase in airway secretions.

Rib fractures and pneumonia were diagnosed by two independent emergency physicians with $>8$ years of experience. Any diagnostic disagreements were resolved by consensus between the physicians.

In this study, the term flail chest was defined as three or more consecutive rib fractures, in two or more locations, creating a flail segment [16].

\section{Post-resuscitation care}

Patients were managed with sedation, analgesia, and mechanical ventilator support according to our standardised protocol, which adheres to resuscitation guidelines [23]. TH was indicated for comatose survivors. The exclusion criterion for TH was shock despite vasopressor use (systolic blood pressure < $90 \mathrm{mmHg}$ ). A central venous catheter and a gastric tube were placed in all patients who underwent $\mathrm{TH}$. The core body temperature was maintained at $33^{\circ} \mathrm{C}$ for $24 \mathrm{~h}$, subsequently rewarmed at a rate of $0.25-$ $0.5^{\circ} \mathrm{C}$ per hour, and maintained at $37^{\circ} \mathrm{C}$ for a further $24 \mathrm{~h}$ with an Arctic Sun ${ }^{\circledR}$ Temperature Management System (Bard BD, Covington, GA, USA).

\section{Statistical methods}

Categorical and continuous variables are expressed as $\mathrm{n}(\%)$ and median (interquartile range), respectively. The diagnostic agreement rate for multiple rib fractures using CT examination was evaluated using the kappa value. Owing to the low number of enrolled patients with the outcome of interest, we conducted the analysis using propensity score matching to adjust for observed confounding factors. To calculate the propensity score, we constructed a logistic regression model including risk factors for the development of pneumonia as independent variables, such as age, sex, total CPR time [24], mechanical ventilation [11-13], TH [25], and use of muscle relaxants [26] and prophylactic antibiotics [13, 27]. Age and total CPR time were categorised ( $\geq 75$ years and CPR time duration over its median value or less). Initially, we evaluated the longitudinal association between the presence of multiple rib fractures and the subsequent incidence of pneumonia using hazard ratios (HRs) adjusted for the propensity score based on a proportional model.

Next, we performed one-to-one propensity score matching between the multiple rib fracture and nonmultiple rib fracture groups. Using a calliper matching method, each patient in the multiple rib fracture group was matched with a patient in the non-multiple rib fracture group without replacement, with the closest estimated propensity within a calliper ( $\leq 0.5$ of the pooled standard deviation of propensity scores). The C-statistic was calculated to evaluate the goodness of fit. We examined the balance in baseline variables between the propensity-matched pneumonia and pneumonia-free groups using standardised differences, with $>10 \%$ indicating imbalance. Furthermore, we compared the period until the 
onset of pneumonia between the propensity score-matched groups using the Kaplan-Meier method and estimated the $p$-value based on the stratified log-rank test. A p-value $<0.05$ denoted statistically significant difference. R version 4.0.2 (R Foundation for Statistical Computing, Vienna, Austria) was used for the statistical analysis.

\section{Results}

During the study period, 683 patients with OHCA were hospitalised after resuscitation. Initially, we excluded patients aged $<18$ years and with CA following trauma. Next, patients who expired within $48 \mathrm{~h}$ and who had confirmed pre-existing pneumonia through chest CT at hospital arrival were excluded. We further excluded 12 patients with missing time data or who did not undergo chest CT. Finally, 87 patients were included in the study analysis (Fig. 1).

Risk factors for pneumonia at baseline in the study participants before and after propensity score matching are shown in Table 1. The diagnostic agreement rate for multiple rib fractures was kappa: 0.81 (95\% confidence interval [Cl]: 0.68-0.93). Thirty-five patients (40.2\%) developed pneumonia within 7 days after hospitalisation. Among all patients before matching $(n=87)$, we found $\geq 0.1$ standardised difference in age, sex, duration of CPR, mechanical ventilation, $\mathrm{TH}$, and use of muscle relaxants. After propensity score matching, we failed to find $\geq 0.1$ standardised difference in any risk factor for pneumonia. The C-statistic was 0.76 (95\% Cl: $0.65-0.86)$ in the model for the calculation of propensity scores.

The unadjusted HR for pneumonia due to multiple rib fractures was significant (HR: 4.81 ; 95\% Cl: $2.35-$ 9.83; $\mathrm{p}<0.001)$. Even after adjustment for propensity scores, the HR for pneumonia remained significant (adjusted HR: 3.51; 95\% Cl: 1.59-7.72; $\mathrm{p}=0.002$ ) (Table 2).

After propensity score matching, the multiple rib fracture group showed significantly shorter pneumoniafree survival than the non-multiple rib fracture group ( $p<0.01$ ) (Fig. 2).

\section{Discussion}

The frequency of rib fractures in this study was $48.3 \%$, and most were bilateral, multiple, and severe. By analysing the propensity score-matched cohort, we found that multiple rib fractures after successful resuscitation were associated with the development of pneumonia within 7 days after hospital admission independent of other risk factors for pneumonia. To the best of our knowledge, while numerous studies have reported on rib fracture caused by chest compression [5-8] and the development of pneumonia after resuscitation [9-15], none have ever addressed this issue.

As the result of a dedicated effort to improve the survival outcome of OHCA, a significant number of resuscitated OHCA patients may develop pneumonia following multiple rib fractures. The management of pneumonia during the post-resuscitation period is critical. Pneumonia can prolong the duration of mechanical ventilation and length of intensive care unit stay, and may worsen the prognosis [12]. Several 
studies have reported various factors associated with the development of pneumonia, such as mechanical ventilation, TH, use of muscle relaxants, and lack of prophylactic antibiotics [9-15]. However, these factors are commonly present in resuscitated patients, as in our study participants. When the study participants were compared according to the presence or absence of multiple rib fractures before propensity score matching, the aforementioned factors were more frequently observed in the multiple rib fracture group. Because the incidence of pneumonia was also higher in this group, it appears that these risk factors were related to the development of pneumonia. However, after adjustment for these factors using propensity scores, our analysis indicated that multiple rib fractures were independently associated with the development of pneumonia during the post-resuscitation period.

The mechanisms through which rib fractures cause pneumonia are generally explained by pain [28], decreased vital capacity [29], and changes in chest wall dynamics that distort the movement of chest wall muscle [30]. The frequency of pneumonia increases with the number of rib fractures, particularly in elderly patients [26] and those with frail chest injuries [27]. Most of the rib fractures observed in this study were bilateral and multiple, and these patients were likely to develop pneumonia within 7 days after hospitalisation. To optimise post-resuscitation care, serious chest wall injuries (e.g., multiple rib fractures) should be carefully evaluated in resuscitated CA patients.

Early surgical fixation of multiple rib fractures or flail chest due to chest trauma improves survival by shortening the duration of mechanical ventilation [19, 20,31]. Similarly, early surgical fixation of fractured ribs can benefit post-resuscitated OHCA patients who experience pneumonia following CPR-related chest wall injuries. Because it is almost impossible to eliminate CPR-related chest wall injuries without compromising the survival of patients with sudden OHCA, physicians need to consider this CPRassociated complication when treating resuscitated patients with OHCA to optimise post-resuscitation care. Further investigation of this issue will reveal its impact on communities and assist in establishing a surgical indication for CPR-related rib fractures.

Our study has several limitations. Firstly, the study was conducted in a single institution over a short observation period to minimise the heterogeneity of the study population. The 2015 resuscitation guideline was applied to our study. In addition, the diagnostic criteria for rib fractures and pneumonia may differ among institutions or investigators. For this reason, we had to establish the diagnostic criteria for this study. Consequently, the sample size is small, and generalization of the present is difficult. Hence, a multicentre study under the same setting is warranted. Secondly, due to the small number of study participants, we were unable to adjust for many confounders using multivariate analysis. Therefore, these findings were obtained through calculation of propensity scores using variables with a small number of risk factors. Although we examined as many risk factors for the development of pneumonia as possible, the possibility of unknown confounding factors cannot be completely ruled out. Differences in patient background between the two groups after propensity score matching were assessed using standardized differences. Although the factors for witnessed and initial shockable rhythm were not fully adjusted for, the lack of difference in the duration of CPR is not expected to have a significant impact on the results of this study. Thirdly, clinical findings (e.g., pain) may not be reflected in the diagnosis of rib fractures 
because we studied sedated patients after resuscitation and evaluated only imaging findings. However, with careful diagnosis, the frequency of rib fractures in the present study was similar to that observed in other studies [7].

\section{Conclusion}

This study using propensity score-matching showed that rib fractures caused by successful resuscitation increased the incidence of pneumonia after hospitalisation. Consideration of the development of pneumonia in OHCA patients with chest wall injury may optimise post-resuscitation care.

\section{Abbreviations}

CA

Cardiac arrest; Cl:Confidence interval; CPR:Cardiopulmonry resuscitation; HR:Hazard ratio; OHCA:Out-ofhospital cardiac arrest; TH:Therapeutic hypothermia

\section{Declarations}

\section{Ethics approval and consent to participate}

The study protocol was approved by the research ethics committees of the Nara Medical University, Japan (decision no. 2328). Given the retrospective observational design of the study, the requirement for participant consent was waived.

\section{Consent for publication}

Not applicable.

\section{Availability of data and materials}

The datasets used and/or analysed during the current study are available from the corresponding author upon reasonable request.

\section{Competing interests}

The authors declare that they have no competing interests.

\section{Funding}

The authors have no financial support to declare.

\section{Authors' contributions}


YK designed the study, collected and analysed the data, and prepared the manuscript. HF revised the manuscript. KS provided support for the analysis of the data. KT, KM, KY, and YT assisted with data collection and critically revised the manuscript. All other authors actively reviewed and approved the final manuscript.

\section{Acknowledgements}

The authors thank the nursing and medical ICU team at the Department of Emergency and Critical Care Medicine of Nara Medical University for their cooperation and support.

\section{Authors' information}

${ }^{1}$ Department of Emergency and Critical Care Medicine, Nara Medical University, Nara, Japan.

${ }^{2}$ Department of Epidemiology, Nara Medical University, Nara, Japan.

\section{References}

1. Perkins GD, Handley AJ, Koster RW, et al. European Resuscitation Council Guidelines for Resuscitation 2015: section 2. Adult basic life support and automated external defibrillation. Resuscitation. 2015;95:81-99.

2. Kleinman ME, Brennan EE, Goldberger ZD, et al. Part 5: adult basic life support and cardiopulmonary resuscitation quality: 2015 American Heart Association guidelines update for cardiopulmonary resuscitation and emergency cardiovascular care. Circulation. 2015;132:S414-35.

3. Vadeboncoeur T, Stolz U, Panchal A, et al. Chest compression depth and survival in out-of-hospital cardiac arrest. Resuscitation. 2014;85:182-8.

4. Stiell IG, Brown SP, Christenson J, et al. What is the role of chest compression depth during out-ofhospital cardiac arrest resuscitation? Crit Care Med. 2012;40:1192-8.

5. Kim EY, Yang HJ, Sung YM, et al. Multidetector CT findings of skeletal chest injuries secondary to cardiopulmonary resuscitation. Resuscitation. 2011;82:1285-8.

6. Hellevuo H, Sainio M, Nevalainen R, et al. Deeper chest compression - more complications for cardiac arrest patients? Resuscitation. 2013;84:760-5.

7. Beom JH, You JS, Kim MJ, et al. Investigation of complications secondary to chest compressions before and after the 2010 cardiopulmonary resuscitation guideline changes by using multi-detector computed tomography: a retrospective study. Scand J Trauma Resusc Emerg Med. 2017;25:8.

8. Yamaguchi R, Makino Y, Chiba F, et al. Frequency and influencing factors of cardiopulmonary resuscitation-related injuries during implementation of the American Heart Association 2010 Guidelines: a retrospective study based on autopsy and postmortem computed tomography. Int $J$ Legal Med. 2017;131:1655-63.

9. Woo JH, Lim YS, Yang HJ, et al. Factors associated with pneumonia in post-cardiac arrest patients receiving therapeutic hypothermia. Am J Emerg Med. 2014;32:150-5. 
10. Kakavas S, Mongardon N, Cariou A, Gulati A, Xanthos T. Early-onset pneumonia after out-of-hospital cardiac arrest. J Infect. 2015;70:553-62.

11. Johnson NJ, Carlbom DJ, Gaieski DF. Ventilator management and respiratory care after cardiac arrest: oxygenation, ventilation, infection, and injury. Chest. 2018;153:1466-77.

12. Perbet S, Mongardon N, Dumas F, et al. Early-onset pneumonia after cardiac arrest: characteristics, risk factors and influence on prognosis. Am J Respir Crit Care Med. 2011;184:1048-54.

13. François B, Cariou A, Clere-Jehl R, et al. Prevention of early ventilator-associated pneumonia after cardiac arrest. N Engl J Med. 2019;381:1831-42.

14. Kim YM, Youn CS, Kim SH, et al. Adverse events associated with poor neurological outcome during targeted temperature management and advanced critical care after out-of-hospital cardiac arrest. Crit Care. 2015;19:283-96.

15. Pabst D, Römer S, Samol A, Kümpers P, Waltenberger J, Lebiedz P. Predictors and outcome of earlyonset pneumonia after out-of-hospital cardiac arrest. Respir Care. 2013;58:1514-20.

16. Dehghan N, de Mestral C, McKee MD, Schemitsch EH, Nathens A. Flail chest injuries: a review of outcomes and treatment practices from the National Trauma Data Bank. J Trauma Acute Care Surg. 2014;76:462-8.

17. Jacobs I, Nadkarni V, Bahr J, et al. Cardiac arrest and cardiopulmonary resuscitation outcome reports: update and simplification of the Utstein templates for resuscitation registries: a statement for healthcare professionals from a task force of the International Liaison Committee on Resuscitation (American Heart Association, European Resuscitation Council, Australian Resuscitation Council, New Zealand Resuscitation Council, Heart and Stroke Foundation of Canada, InterAmerican Heart Foundation, Resuscitation Councils of Southern Africa). Circulation. 2004;110:3385-97.

18. Dunham GM, Perez-Girbes A, Bolster F, Sheehan K, Linnau KF. Use of whole body CT to detect patterns of CPR-related injuries after sudden cardiac arrest. Eur Radiol. 2018;28:4122-7.

19. Kasotakis G, Hasenboehler EA, Streib EW, et al. Operative fixation of rib fractures after blunt trauma: a practice management guideline from the Eastern Association for the Surgery of Trauma. J Trauma Acute Care Surg. 2017;82:618-26.

20. Beks RB, Peek J, de Jong MB, et al. Fixation of flail chest or multiple rib fractures: current evidence and how to proceed. A systematic review and meta-analysis. Eur J Trauma Emerg Surg. 2019;45:631-44.

21. Kralj E, Podbregar M, Kejžar N, Balažic J. Frequency and number of resuscitation related rib and sternum fractures are higher than generally considered. Resuscitation. 2015;93:136-41.

22. Nielsen N, Sunde K, Hovdenes J, et al. Adverse events and their relation to mortality in out-of-hospital cardiac arrest patients treated with therapeutic hypothermia. Crit Care Med. 2011;39:57-64.

23. Soar J, Callaway CW, Aibiki M, et al. Part 4: advanced life support: 2015 International Consensus on Cardiopulmonary Resuscitation and Emergency Cardiovascular Care Science with Treatment Recommendations. Resuscitation. 2015;95:e71-120. 
24. Kashiwagi Y, Sasakawa T, Tampo A, et al. Computed tomography findings of complications resulting from cardiopulmonary resuscitation. Resuscitation. 2015;88:86-91.

25. Mongardon N, Perbet S, Lemiale V, et al. Infectious complications in out-of-hospital cardiac arrest patients in the therapeutic hypothermia era. Crit Care Med. 2011;39:1359-64.

26. Lascarrou JB, Le Gouge A, Dimet J, et al. Neuromuscular blockade during therapeutic hypothermia after cardiac arrest: observational study of neurological and infectious outcomes. Resuscitation. 2014;85:1257-62.

27. Davies KJ, Walters JH, Kerslake IM, Greenwood R, Thomas MJ. Early antibiotics improve survival following out-of hospital cardiac arrest. Resuscitation. 2013;84:616-9.

28. Liu T, Liu P, Chen J, et al. A randomized controlled trial of surgical rib fixation in polytrauma patients with flail chest. J Surg Res. 2019;242:223-

29. Carver TW, Milia DJ, Somberg C, Brasel K, Paul J. Vital capacity helps predict pulmonary complications after rib fractures. J Trauma Acute Care Surg. 2015;79:413-6.

30. Talbot BS, Gange CP Jr, Chaturvedi A, Klionsky N, Hobbs SK, Chaturvedi A. Traumatic rib injury: patterns, imaging pitfalls, complications, and treatment. Radiographics. 2017;37:628-51.

31. Kocher GJ, Sharafi S, Azenha LF, Schmid RA. Chest wall stabilization in ventilator-dependent traumatic flail chest patients: who benefits? Eur J Cardiothorac Surg. 2017;51:696-701.

\section{Tables}

Due to technical limitations, table 1-2 is only available as a download in the Supplemental Files section.

\section{Figures}




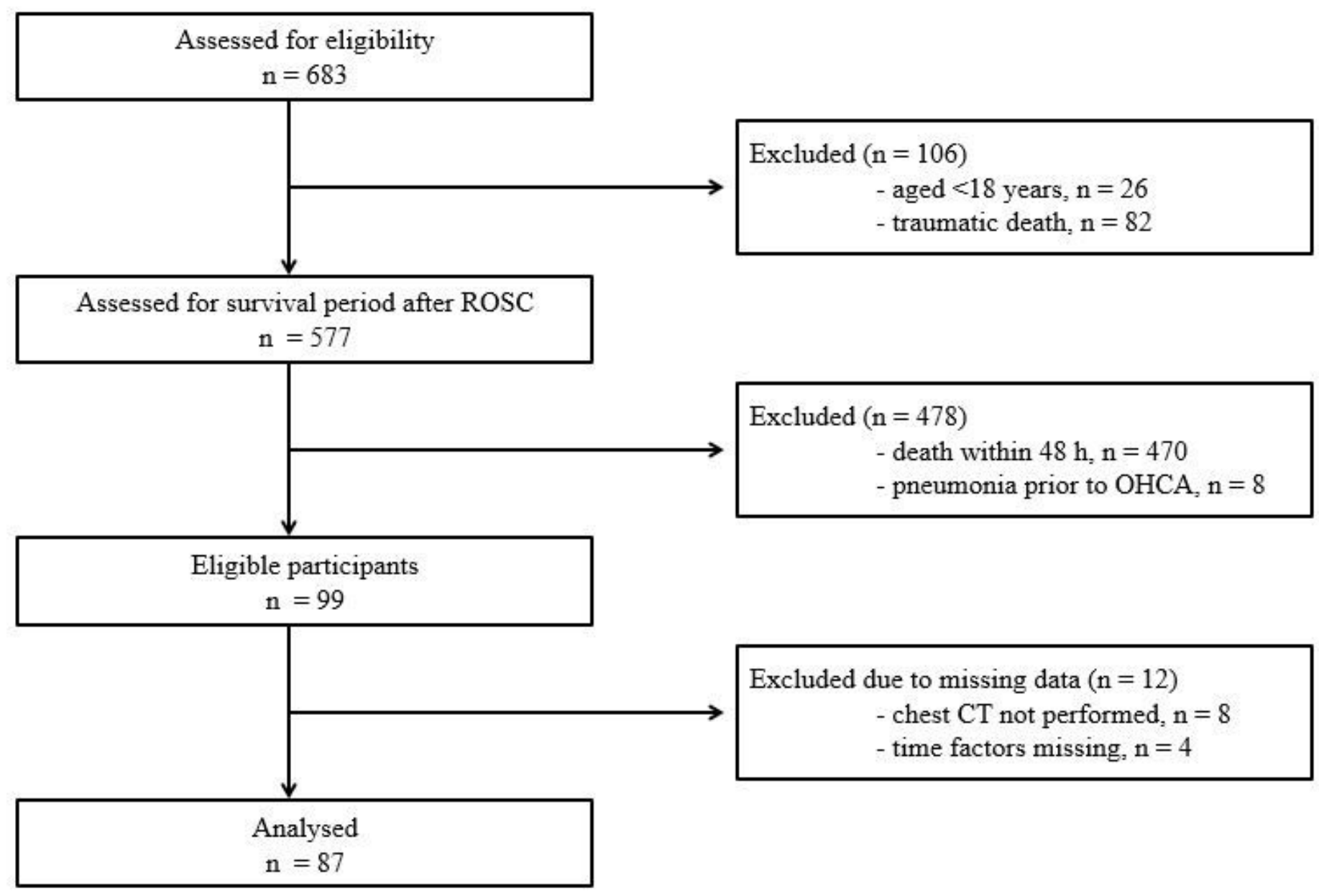

\section{Figure 1}

Flowchart of participants. CT, computed tomography; OHCA, out-of-hospital cardiac arrest; ROSC, return of spontaneous circulation 


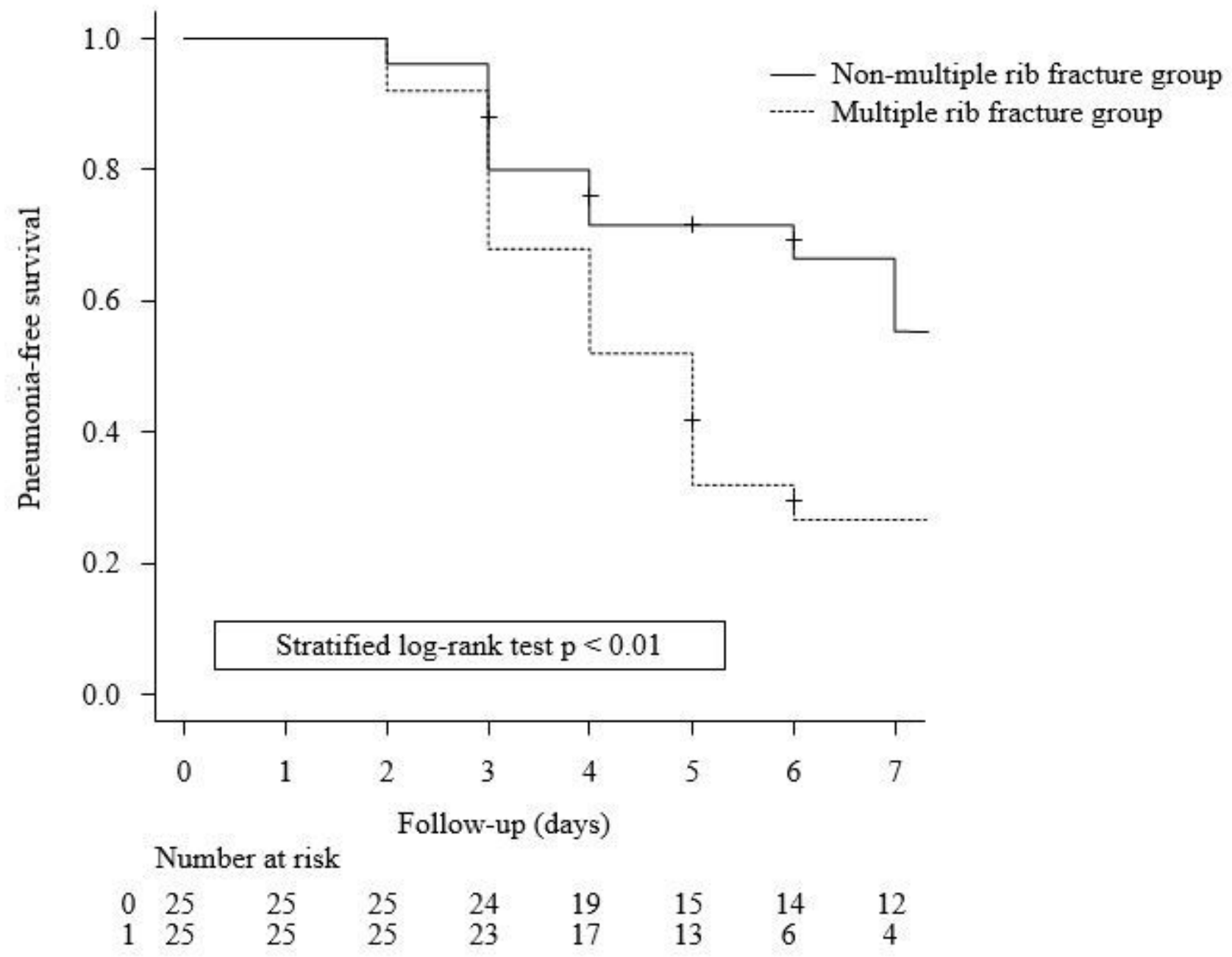

Figure 2

Kaplan-Meier pneumonia-free curves: multiple rib fracture group versus non-multiple rib fracture group.

\section{Supplementary Files}

This is a list of supplementary files associated with this preprint. Click to download.

- Tab1.xlsx

- Tab2.xlsx 